\title{
A Tailored Strategy for Recipient Hepatectomy: Left Portal Flow Preserving High Hilar Dissection
}

\author{
Ahmed Shehta ${ }^{1,2} \cdot$ Jaehong Jeong ${ }^{1,3} \cdot$ Kwang-Woong Lee $^{1} \cdot$ Jeong-Moo Lee ${ }^{1}$ Suk Kyun Hong ${ }^{1} \cdot$ Jae-Hyung Cho ${ }^{1}$. \\ Nam-Joon $\mathrm{Yi}^{1} \cdot$ Kyung-Suk Suh ${ }^{1}$
}

Received: 6 March 2019 / Accepted: 18 May 2019 / Published online: 16 July 2019

(C) 2019 The Society for Surgery of the Alimentary Tract

\begin{abstract}
Background Lee et al. (Liver Transpl 10(9):1158-1162, 2004) reported high hilar dissection (HHD) technique as a novel method for recipient hepatectomy to overcome limitations of conventional hilar dissection (CHD). HHD allowed performing multiple tension-free biliary anastomoses and easy reconstruction of double portal vein orifices. However, longer anhepatic phase is its main drawback. We describe a new modification of original HHD, called left portal vein flow preserving HHD (LFP-HHD). The new technique aims to gain the balance between CHD and original HHD.

Methods The detailed technique of recipient hepatectomy by LFP-HHD is shown in the electronic video file. It involves high intrahepatic division of hilar structures while maintaining portal drainage through maintained left portal vein (LPV). Control of right hemi-liver inflow allows for division of right hepatic vein, and safe dissection of inferior vena cava (IVC) and hilar structures. According to coordination with donor surgery, the liver could be easily explanted.

Discussion LFP-HHD has the same principle of original HHD allowing for multiple tension-free well-vascularized biliary anastomoses. LFP-HHD allows for shortening of anhepatic phase duration as portal venous drainage is continued through maintained LPV avoiding prolonged total portal clamping with bowel edema or the need for temporary porto-caval shunt. On the other hand, the number and the length of the hilar structures can be decreased compared with the original HHD technique. However, the level of division of the hilar structures is acceptable in most of the cases.

Conclusion LFP-HHD is a novel simple technique for recipient hepatectomy that can be tailored for certain clinical conditions.
\end{abstract}

Keywords Modified high hilar dissection $\cdot$ Living donor liver transplantation $\cdot$ Recipient hepatectomy

Ahmed Shehta and Jaehong Jeong contributed equally to this work as cofirst authors.

Electronic supplementary material The online version of this article (https://doi.org/10.1007/s11605-019-04281-2) contains supplementary material, which is available to authorized users.

Kwang-Woong Lee

kwleegs@gmail.com

1 Department of Surgery, College of Medicine, Seoul National University, 28 Yongon-Dong, Chongno-gu, Seoul 110-744, South Korea

2 Liver Transplantation Unit, Gastrointestinal Surgery Center, College of Medicine, Mansoura University, Mansoura, Egypt

3 Department of Surgery, School of Medicine, Soonchunhyang University, Bucheon, South Korea

\section{Compliance with Ethical Standards}

Conflict of Interest The authors declare that they have no conflicts of interest.

\section{Reference}

1. Lee KW, Joh JW, Kim SJ, et al. High hilar dissection: new technique to reduce biliary complication in living donor liver transplantation. Liver Transpl 2004;10(9):1158-1162.

Publisher's Note Springer Nature remains neutral with regard to jurisdictional claims in published maps and institutional affiliations. 